\title{
Terminologie des indices boursiers
}

\section{Michel Van der Yeught}

\section{OpenEdition}

\section{Journals}

Édition électronique

URL : http://journals.openedition.org/asp/3512

DOI : 10.4000/asp.3512

ISSN : 2108-6354

\section{Éditeur}

Groupe d'étude et de recherche en anglais de spécialité

\section{Édition imprimée}

Date de publication : 1 décembre 1996

Pagination : 207-216

ISSN : 1246-8185

\section{Référence électronique}

Michel Van der Yeught, «Terminologie des indices boursiers », ASp [En ligne], 11-14 | 1996, mis en ligne le 08 mai 2013, consulté le 01 mai 2019. URL : http://journals.openedition.org/asp/3512 ; DOI : 10.4000/asp.3512

Ce document a été généré automatiquement le 1 mai 2019.

Tous droits réservés 


\title{
Terminologie des indices boursiers
}

\author{
Michel Van der Yeught
}

1 Les enseignants d'anglais des affaires font fréquemment référence aux indices boursiers. Le Dow Jones, le Nikkeï, le CAC-40 sont les indicateurs financiers les plus cités et ils sont en général familiers à nos étudiants. Pourtant, cette familiarité est très superficielle et on ignore généralement ce que sont les indices et comment ils fonctionnent. De même, on ne soupçonne souvent pas qu'ils sont beaucoup plus nombreux que les quelques indices vedettes quotidiennement mentionnés par les médias. Sur le plan linguistique, l'enseignant d'anglais de spécialité doit en savoir un peu plus que ses étudiants s'il veut éviter de confondre index et average, FT 30 et FT-SE 100 et s'il souhaite pouvoir expliquer pourquoi un article fait tantôt référence au DJIA tantôt au S\&P 500.

2 Le présent article se propose de faire le point sur les grands indices boursiers britanniques et américains sans pour autant prétendre à l'exhaustivité ni à la spécialisation boursière. Il ne s'adresse pas à des professionnels des marchés mais à des linguistes. Leur travail n'est pas de prendre des positions en fonction des indicateurs boursiers mais d'en savoir suffisamment sur ceux-ci pour en comprendre la terminologie anglaise, et pour produire eux-mêmes un anglais ou un français qui «colle » bien à la réalité. C'est ainsi qu'ils analyseront en finesse un article du Wall Street Journal ou qu'ils traduiront dans un anglais précis une page du Journal des Finances.

\section{Indexes ou indices?}

Pour le mot index deux pluriels existent : indexes et indices. Les dictionnaires sont de peu de recours pour choisir le bon pluriel. Certains donnent les deux, d'autres un seul, l'un ou l'autre. Une lecture suivie de la presse financière anglo-américaine montre pourtant qu'en règle générale, les Nord-Américains écrivent indexes ${ }^{1}$ et les Britanniques indices ${ }^{2}$.

4 Il conviendra donc d'adapter les pluriels au contexte national et de les homogénéiser lors de la production d'anglais. 


\section{Index ou average?}

$5 \mathrm{Au}$ seul terme français "indice» correspondent deux termes anglais index et average. Même si la différence entre les deux ne peut être rendue en français, il convient d'en tenir compte pour pouvoir l'expliquer en cours et pour sélectionner à bon escient l'un ou l'autre terme lors de la production d'anglais.

6 Le terme le plus ancien est average. Il peut être rendu par « moyenne boursière » car c'est en effet ce qu'il était à l'origine. Lorsque Charles Dow calcula son premier indice en 1884, il additionna le cours de onze importantes valeurs et en fit la moyenne en divisant le total par onze. Le terme average a perduré mais il ne correspond précisément qu'à des indices calculés à partir d'une moyenne arithmétique simple. À l'heure actuelle, la plupart des averages sont les indices calculés par la société américaine Dow Jones \& Co. et par l'hebdomadaire boursier Barron's, lui-même édité par cette même société.

7 Il faut noter que si les indices Dow Jones sont de simples moyennes, leur calcul s'est compliqué au fil des années. D'une part leur composition s'est généralement étoffée (le Dow Jones Industrial Average par exemple est passé de onze à trente valeurs); d'autre part, la moyenne a dû tenir compte de divers facteurs de distorsion des résultats par exemple les divisions de titres (stock split). Un nombre régulièrement ajusté (le divisor) permet de compenser l'effet de ces distorsions et de préserver la continuité représentative de l'indice. La presse américaine spécialisée publie régulièrement les " diviseurs » actualisés des moyennes boursières Dow Jones.

Le terme index désigne des indices qui calculent la moyenne des cours par rapport à une valeur de base du marché (base value) à un moment donné (base date). Le Standard \& Poor's 500 (S\&P 500), par exemple, marque l'évolution du cours de 500 grandes sociétés américaines par rapport à une moyenne des cours fixée à dix dollars sur la période de base 1941-1943. Le FT-SE 100 britannique indique l'évolution des cours des cent premières sociétés cotées à la Bourse de Londres par rapport à une valeur de base de 1000 points au 30 décembre 1983. Certains indices sont calculés en unités monétaires (le dollar par exemple), mais la plupart sont calculés en points. Un seul indice Dow Jones n'est pas une moyenne mais bien un index. Il s'agit du Dow Jones Equity Market Index qui mesure l'évolution d'environ 700 titres par rapport à une valeur de 100 points au 30 juin 1982.

Dans la pratique, les averages, qui sont un sous-ensemble des indices, sont fréquemment appelés indexes ${ }^{3}$. Mais l'inverse n'est pas vrai et il faut éviter d'employer le mot average pour désigner un indice qui n'est pas une moyenne boursière dépourvue de date et de valeur de base. Le français, qui exprime une expérience plus récente des indices boursiers que l'anglo-américain ne peut restituer la nuance index/average, sauf à faire suivre le mot indice d'une explication périphrastique.

\section{Indices larges ou étroits?}

10 Un indice est jugé étroit (narrow) lorsqu'un nombre réduit de valeurs entre dans sa composition. C'est le cas du Dow Jones des valeurs industrielles (DJIA) qui ne comporte que trente valeurs. En revanche, plus un indice inclut de valeurs plus il est jugé large ( broad ou broad-based) et représentatif (representative) des évolutions de l'ensemble du marché. 
11 Les indices larges ne sont pourtant pas nécessairement ceux que les investisseurs utilisent le plus. Le nombre élevé des sociétés suivies rend leur calcul trop lent pour les opérateurs en quête d'indicateurs de tendance instantanés. C'est ainsi que l'indice Wilshire 5000 qui suit plus de 6000 sociétés américaines n'est calculé qu'une fois par jour alors que la plupart des autres indices sont calculés en temps réel (toutes les 30 secondes ou toutes les minutes). En outre, les indices larges retardent et estompent les ruptures d'évolutions alors que les indices étroits les accentuent et permettent aux opérateurs de mieux suivre la tendance ou de l'anticiper.

Les grands indices de référence sont donc ceux qui combinent le mieux la représentativité et l'immédiateté. Le succès du S\&P 500 s'explique par le fait qu'il est plus représentatif $d u$ marché américain que le très étroit Dow Jones, tout en indiquant finement et en temps réel ses moindres évolutions. De même, les responsables britanniques ont lancé le FT-SE 100 en 1984 pour remplacer le FT 30 étroit et daté (il avait été créé en 1935).

\section{Indices généraux, « composites » ou spécialisés?}

13 Aux notions d'étroitesse ou de largeur des indices s'ajoute le problème de la sélection des valeurs qui les composent.

Certains indices suivent la totalité ou la quasi-totalité des titres cotés sur un marché tous secteurs confondus. C'est par exemple le cas du FT-SE Actuaries All-Share qui suit plus de 900 sociétés cotées à Londres. Le terme all-share, que l'on peut rendre par " général », est d'ordinaire utilisé en Grande-Bretagne. Les Américains lui préfèrent le terme composite quoiqu'il y ait, à strictement parler, une différence entre les deux termes. En l'absence d'équivalent direct en français, il faut comprendre composite dans le sens de «tous secteurs confondus » ou "à échantillon représentatif ». Certains indices "composites » suivent l'ensemble d'un marché donné et sont donc également des indices généraux ou all-share dans la terminologue britannique. C'est le cas du New York Composite Index qui suit tous les titres cotés au New York Stock Exchange. D’autres indices « composites » ne suivent qu'un échantillon, réduit, mais représentatif, d'un marché. C'est le cas du S\&P 500 également appelé S\&P Composite Index.

Les indices généraux et « composites » se décomposent souvent en sous-indices (subindex) spécialisés qui suivent un secteur d'activité particulier (valeurs des transports, valeurs financières, services publics) ou des sociétés de taille similaire (blue chips, PME, etc.).

16 C'est ainsi que l'indice Dow Jones Composite (également appelé Dow Jones 65 Stock Average) se décompose en :

- Dow Jones Industrial Average (30 valeurs industrielles),

- Dow Jones Transportation Average (20 valeurs des transports),

- Dow Jones Utility Average(15 valeurs des services publics).

Il apparaît ainsi que l'indice Dow Jones universellement connu n'est qu'un indice spécialisé dans les grandes valeurs industrielles. Les grandes firmes de services comme Walt Disney (médias, cinéma) et J.P. Morgan (banque) n’y ont été admises qu'en 1991.

De même, le FT-SE A 350 britannique résulte de la combinaison du FT-SE 100 (les cent premières blue chips du Royaume-Uni) et du FT-SE Mid-250 (250 sociétés de capitalisation 
moyenne). Par ailleurs, le FT-SE SmallCap suit toutes les PME du FT-SE A All-Share qui ne figurent pas dans le FT-SE 350.

\section{Indices indicateurs boursiers et indices « objets de marché »}

La fonction la plus connue des indices est d'être des indicateurs de tendances boursières. Dans les années 1980, cependant, le développement rapide des marchés et des techniques a fait des indices eux-mêmes des supports d'investissement. Au lieu de passer du temps à se constituer des portefeuilles rentables et équilibrés, de nombreux investisseurs choisirent d'acheter la totalité d'un indice donné qui devenait par là même un produit financier. Des options et des contrats à terme sur indice se sont rapidement développés et par voie de conséquence de nombreux indices nouveaux ont été construits, non pour servir d'indicateurs mais pour satisfaire aux diverses exigences de l'investissement sur indice. Les boursiers disent que ces indices sont devenus le «sous-jacent » de contrats d'investissement et ils les appellent indices "objets-de-marché »"

Le praticien d'anglais de spécialité doit donc adapter son analyse ou sa traduction à cette nouvelle réalité et éviter de qualifier un indice objet-de-marché d'indicateur boursier, et vice-versa.

En bref, trois cas de figure peuvent se présenter :

22 1. L'indice est exclusivement un indicateur boursier. C'est le cas des indices Dow Jones, la société Dow Jones \& Co. ayant toujours refusé que ses indices fassent l'objet de contrats d'investissement.

2. L'indice est à la fois indicateur et objet d'investissement. C'est généralement le cas des grands indices de référence (S\&P 500, FT-SE 100, CAC-40, etc.).

3. L'indice a été exclusivement créé à des fins d'investissement et n'a pas, ou peu, de valeur d'indicateur. Par exemple, le S\&P 100 créé pour les options ; l'AMEX Major Market Index (MMI) créé pour suivre les mouvements du Dow Jones tout en faisant l'objet de contrats à terme et de contrats d'options, etc.

\section{Indices pondérés par les prix ou par la capitalisation boursière?}

Un indice est dit price-weighted (pondéré par les cours) lorsque les cours les plus élevés influencent davantage son évolution que les cours les plus bas. Les averages Dow Jones ainsi que le Nikkeï japonais sont de ce type. Cette catégorie d'indices tend à devenir obsolète parce qu'elle n'intègre pas la notion de capitalisation boursière, c'est-à-dire le nombre d'actions cotées sur le marché pour une valeur donnée. Il peut ainsi se produire que les mouvements d'une valeur chère mais à faible nombre d'actions influencent davantage l'indice qu'une valeur peu chère mais à nombre élevé d'actions.

Un indice est dit market value-weighted ou capitalization-weighted (pondéré par la capitalisation boursière) quand son calcul intègre la notion de capitalisation boursière (le cours de l'action multiplié par le nombre d'actions). Dans cette classe d'indices, les valeurs fortement représentées sur le marché influencent largement l'indice, même si la 
variation de leur cours est faible. La plupart des grands indices modernes (S\&P 500, FT-SE 100, CAC-40, etc.) à l'exception du Nikkeï, sont de ce type.

\section{Sur quelques erreurs et confusions}

$$
\text { confusions que les remarques suivantes permettront d'identifier. }
$$

\section{Dow Jones}

En France, l'indice et la société Dow Jones sont souvent orthographiés avec un trait d'union. Dow Jones n'en prend pas.

\section{Standard \& Poor's}

Le nom de la grande agence américaine de notation financière qui publie les indices S\&P 500 , S\&P 100, etc., est souvent mal orthographié. On trouve fréquemment «Standard \& Poor » ou «Standard \& Poors ». Éviter également d'écrire Standard and Poor's; préférer l'esperluette.

\section{FT 30 et FT-SE 100}

La plupart des indices publiés par le Financial Times sont calculés en collaboration avec la Bourse de Londres (London Stock Exchange). Ils en tirent leur sigle FT-SE (Financial Times-Stock Exchange). Un indice fait cependant exception à la règle, le vieux FT 30 (également appelé Financial Times Ordinary Share Index) publié depuis 1935 par le journal. Il ne faut donc pas écrire « FT-SE 30 ».

\section{Footsie}

31 Par ailleurs, le nom familier donné à l'indice FT-SE 100 vient de l'assimilation phonétique FT-SE/footsie (to play footsie, faire du pied) et ne s'applique qu'à cet indice. Le FT 30, dont le sigle ne comporte pas les lettres SE, ne peut donc pas s'appeler « Footsie » comme on le lit souvent.

\section{Conclusion}

Il n'y a pas de différence technique entre les indices boursiers français et anglo-saxons, mais l'anglais et le français sont marqués dans ce domaine par des expériences historiques différentes. La taille du marché boursier américain et la grande diversité des indices qui s'y sont développés expliquent également que certaines notions échappent à la langue française dont l'expérience des indices est relativement récente. Il faut donc rester conscient, lors de la traduction ou l'analyse d'un texte, que le même mot indice peut recouvrir des réalités très diverses qui ne peuvent être négligées ou caricaturées sous peine de contresens. En l'espèce, le problème terminologique des indices boursiers est typique des problèmes de langue de spécialité. D'un pays à l'autre, les techniques se superposent souvent exactement, les langues et les cultures rarement. 


\section{BIBLIOGRAPHIE}

Brown, J. D. 1991. 101 Years on Wall Street. Englewood Cliffs, NJ : Prentice Hall, 221-251.

Downes, J. \& J. E. Goodman. 1991. Dictionary of Finance and Investment Terms. Barron's Educational

Series. New York : Hauppauge.

Gobry, P. 1988. La bourse aux indices. Paris : Économica.

Inman, C. 1994. The Financial Times Style Guide. Londres : Pitman Publishing.

\section{ANNEXES}

\section{Annexe 1}

Mini-glossaire récapitulatif

Average : indice ; moyenne boursière simple.

Base date : date de base; date où la valeur de base d'un indice est fixée et à partir de laquelle l'indice est calculé.

Base value : niveau de départ d'un indice fixé à une date donnée. Ce niveau de départ est souvent (mais pas obligatoirement) un nombre rond :10 points, 50 points, 1000 points, etc.

Capitalization-weighted : pondéré par la capitalisation boursière.

Composite : «composite»; à échantillon représentatif.

Divisor : diviseur d'ajustement.

Index : indice ; le terme recouvre «index» et «average».

Market value-weighted : pondéré par la capitalisation boursière

Price-weighted : pondéré par les cours.

\section{NOTES}

1. Voir par exemple le Wall Street Journal ou le Barron's ainsi que l'entrée index du Dictionary of Finance and Investment Terms de Downes et Goodman, p. 196.

2. Voir par exemple le Financial Times et le chapitre «Stock Market Indices » du Financial Times Style Guide, p. 221-224.

3. Par exemple, l'Amex Major Market Index (MMI) est en réalité un average.

4. Voir le livre de Pascal Gobry essentiellement consacré aux indices objets-de-marché, La Bourse aux indices. 


\section{RÉSUMÉS}

Les indices boursiers sont omniprésents en anglais financier. Les plus importants sont universellement familiers mais leur nature et leur fonctionnement restent largement méconnus. Des distinctions (average/index, narrow index/broad index, price-weighted/market value-weighted, all share/ composite/ subindex) permettront à l'angliciste de spécialité d'adapter à chaque indice la terminologie française ou anglaise qui lui correspond. Des remarques sur des erreurs courantes, un mini-glossaire, une liste des principaux indices étudiés et une bibliographie complètent cette étude.

Stock market indices are frequently mentioned in financial English. The major indices are universally familiar but what they really are and how they work generally remains little understood. Using systematic distinctions (average/index, narrow index/broad index, priceweighted/market value-weighted, all share/composite/subindex) will enable the practitioner of English for special purposes to adopt for each index the appropriate English and French terms. This survey includes remarks on common spelling mistakes and confusions, a mini-glossary, a list of the most important British and American indices and a bibliography.

\section{INDEX}

Mots-clés : anglais de spécialité, Dow Jones, Footsie, indice boursier, langue de la bourse, marché financier

Keywords : English for Special Purposes, financial market, language of the stock exchange, stock market average, stock market index

\section{AUTEUR}

\section{MICHEL VAN DER YEUGHT}

Michel Van der Yeught enseigne l'anglais financier et comptable à l'IUT de Toulon. Il s'est spécialisé dans la lexicographie et la traduction de l'anglais de la bourse.

michel.vanderyeught@univ-amu.fr 